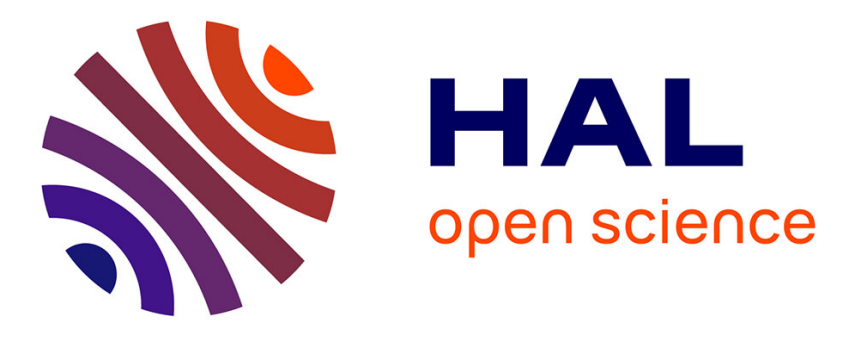

\title{
Esmeraldas, un territoire urbain entre risques et ressources pétrolières
}

Julien Rebotier, Pascale Metzger, Patrick Pigeon

\section{To cite this version:}

Julien Rebotier, Pascale Metzger, Patrick Pigeon. Esmeraldas, un territoire urbain entre risques et ressources pétrolières. Les Cahiers d'Outre-Mer. Revue de géographie de Bordeaux, 2019, 2 (280), pp.469-494. 10.4000/com.10586 . halshs-02908179

\section{HAL Id: halshs-02908179 \\ https://shs.hal.science/halshs-02908179}

Submitted on 21 Jun 2021

HAL is a multi-disciplinary open access archive for the deposit and dissemination of scientific research documents, whether they are published or not. The documents may come from teaching and research institutions in France or abroad, or from public or private research centers.
L'archive ouverte pluridisciplinaire HAL, est destinée au dépôt et à la diffusion de documents scientifiques de niveau recherche, publiés ou non, émanant des établissements d'enseignement et de recherche français ou étrangers, des laboratoires publics ou privés. 


\title{
Citer comme :
}

Rebotier, J., Metzger, P., Pigeon, P., 2019, « Esmeraldas, un territoire urbain entre risques et ressources pétrolières », Cahiers d'Outre-Mer, 72(280), 469-494

\section{Esmeraldas, un territoire urbain entre risques et ressources pétrolières}

Julien Rebotier, julien.rebotier@.cnrs.fr, chargé de recherches au CNRS, LISST UMR 5193

Metzger Pascale, pascale.metzger@ird.fr, chargée de recherches à l'IRD, PRODIG UMR 8586

Patrick Pigeon, patrick.pigeon@usmb-univ.fr, professeur de géographie, Université Savoie Mont-Blanc

\section{Résumé}

À Esmeraldas (Équateur), la présence d'infrastructures liées à l'activité pétrolière recompose à la fois l'urbanisation et les risques associés, en les augmentant et les diversifiant. Simultanément, tant la redistribution de la rente pétrolière que les politiques de prévention ne peuvent ni contrôler ces recompositions urbaines ni réduire les risques associés. L'article remet Esmeraldas dans le contexte géopolitique national et international pour mieux comprendre l'importante fragmentation du jeu d'acteur et une situation qui peut sembler paradoxale. Il propose aussi des pistes, à partir d'une lecture territoriale, qui pourraient répondre à une partie des dysfonctionnements dont sont conscients les acteurs nationaux et locaux.

Mots-clés : Pétrole, risques, gouvernance, territoire, intégration, planification, Équateur.

\section{Esmeraldas, an Urban Area Grappling with Risk and Oil Resources}

\begin{abstract}
In Esmeraldas (Ecuador), the infrastructures related with oil activities transform the urban fabric and the pre-existing risks at the same time. Meanwhile, oil income redistribution as well as existing risk prevention policies do not curb the prevailing trends towards disasters in the making. Prevention policies are still framed according to specific hazards types and highly segmented, driven by specific institutional stakeholders. The national and international contexts are presented, as they help to understand the high degree of fragmentation between actors in such a strategic area. Based on a territorial approach, the paper presents solutions that might contribute to improve a situation the national and local stakeholders are conscious of.
\end{abstract}

Keywords: Oil, risks, governance, territory, integration, planning, Ecuador. 


\section{Introduction}

Le but de cet article est de réfléchir aux relations entre ressources, risques et territoire, à partir de l'exemple d'Esmeraldas, en Équateur. Esmeraldas est la capitale de la province du même nom. Située sur la côte pacifique, à l'embouchure du fleuve Esmeraldas, la ville compte aujourd'hui environ 200000 habitants, principalement afro descendants (Minda, 2015). Il s'agit d'une ville portuaire, caractérisée par un déficit d'équipements et de services urbains, l'auto construction des habitations, l'informalité des activités économiques, et un pouvoir municipal faible (Estupiñán Carvache, 2011).

Dans les années 1970 l'arrivée des activités pétrolières a transformé la dynamique spatiale, les acteurs et les ressources de ce territoire (Collin Delavaud, 1978 ; Jaramillo, 1980 ; CONADE, 1980). Les infrastructures pétrolières situées dans l'aire urbaine d'Esmeraldas sont considérées comme stratégiques pour l'Équateur et constituent des enjeux nationaux de la souveraineté du pays. La présence de ces installations contribue à recomposer la dynamique urbaine d'Esmeraldas, tout autant qu'elle reconfigure les risques et la gouvernance de ce territoire. Ces évolutions préparent clairement de futurs désastres, mais les politiques de gestion des risques sont à la fois limitées et fragmentées : elles ne concernent souvent qu'un type d'aléa, et sont menées en fonction d'un type d'acteur impliqué. Elles sont cohérentes avec la définition classique des risques, qui associent un aléa à des éléments exposés inégalement vulnérables (Pigeon et Rebotier, 2016).

Cette situation incite donc à interroger comment et pourquoi les ressources associées aux hydrocarbures contribuent à augmenter les risques à la fois pour les acteurs nationaux et pour les acteurs locaux, notamment la population d'Esmeraldas. Cet exemple permet, pour reprendre la formulation de l'appel, «d'interroger le risque en le sortant de la dichotomie (aléa/vulnérabilité) des travaux classiques sur les risques ». L'article illustre comment la gestion des ressources contribue « à la fabrique des territoires », et se traduit par l'augmentation et la diversification des risques en fonction des acteurs concernés. Ainsi, le propos s'inscrit à la fois dans la perspective d'une construction sociale des risques (García-Acosta, 2005), portée dans la région par le réseau $L a R^{2} d^{1}$ et incarnée à l'international par le courant dit des « radicaux » (Wisner et al., 1994 ; Oliver-Smith et al. 2017) ; et dans la fabrique des risques c'est-à-dire une

\footnotetext{
${ }^{1}$ www.desenredando.org
} 
lecture sociale et politique des situations de risques cristallisées dans un contexte, en un lieu, et dans une configuration d'acteurs particuliers (Gilbert, 2013).

L'Équateur est un pays qui est, à l'échelle mondiale, peu engagé dans les échanges internationaux de marchandises : sa part dans ces derniers est estimée à $0,11 \%$ seulement en 2017 (OMC, 2019). Toutefois, l'OMC attire l'attention sur l'importance relative de l'exportation et l'importation des hydrocarbures pour l'Équateur, de surcroît membre de l'OPEP, même si les exportations de produits agricoles l'emportent en valeur. Selon l'ambassade de France en Équateur en 2019 : «L'économie équatorienne est dépendante des cours mondiaux du pétrole ( $40 \%$ des revenus de l'État et $60 \%$ des exportations) $»^{2}$.

Le pétrole joue un rôle important dans l'économie nationale depuis les années 1970. Le tournant réformiste de la Révolution Citoyenne porté par Rafael Correa à partir de la constitution de 2008 apporte certes une réaffirmation de l'État et un renforcement de la responsabilité publique, mais sans véritablement rompre avec des pratiques « extractivistes » et « développementistes » (Fontaine, 2006; Ramírez Gallegos, 2010 ; Le Quang et Ramírez Gallegos, 2016). Les revendications ethniques et écologistes du virage à gauche équatorien cèdent progressivement face aux impératifs du développement (Fontaine et Narváez, 2007). Le néo-extractivisme, s'il élargit l'assiette de redistribution de la rente, entérine une forme de prédation environnementale qui persiste dans le temps (Juteau et al., 2014 ; Fontaine, 2015), Dans ces conditions, la ressource pétrolière reste centrale, et l'on comprend pourquoi il reste impératif de « sécuriser la rente pétrolière » dans les arbitrages budgétaires nationaux (Acosta et Albornoz, 2010).

Cette rente ne profite guère aux populations d'Esmeraldas (Jurado, 2006a ; Valdivia, 2018). La situation est dénoncée par un ancien maire d'Esmeraldas (Estupiñán, 2004). La presse locale (La Hora) et nationale (El Comercio, El Telégrafo), aborde régulièrement le sujet de la faiblesse de la redistribution locale de la rente pétrolière, ce d'autant plus qu'une loi de 2012 en réaffirme la nécessité. En outre, les nuisances de l'exploitation pétrolière à Esmeraldas sont largement et diversement documentées, depuis les inconvénients posés par les premiers travaux jusqu'à la pollution diffuse (OIPE, 1975 ; CEPAL, 1990 ; Jurado, 2006b ; Pacheco Luque, 2011 ; Burgos Cisneros, 2016).

La gestion des ressources pétrolières aboutit à la fois à remettre en question l'urbanisation d'Esmeraldas, à augmenter et diversifier les risques, et à réduire la capacité d'agir des pouvoirs locaux. Face à un tel bilan, on peut légitimement se demander pourquoi les politiques de gestion des risques demeurent fragmentées et limitées, ce qui revient à augmenter encore le niveau de risque pour l'aire urbaine d'Esmeraldas comme pour les enjeux nationaux stratégiques qui s'y trouvent. Comprendre une telle situation nécessite de reconstituer le contexte géopolitique qui est celui d'Esmeraldas. Des propositions permettant de faire évoluer cette situation à partir d'une approche par le territoire peuvent être suggérées.

${ }^{2}$ https://www.diplomatie.gouv.fr/fr/dossiers-pays/equateur/presentation-de-l-equateur/ 


\section{Stratégique, la ressource pétrole reconfigure et éclate le territoire}

\subsection{Un secteur stratégique et des acteurs de poids qui s'inscrivent dans l'espace local}

Les infrastructures que nécessitent le transport et les échanges internationaux d'hydrocarbures, comme les activités liées à la production d'énergie qui leur sont associées, sont stratégiques pour l'Équateur. Comme pour tout autre État, elles représentent un enjeu de souveraineté nationale.

À Esmeraldas, le complexe d'installations interdépendantes dans les domaines du transport et de la production d'énergie liés aux hydrocarbures est géré par trois entreprises : PetroEcuador, OCP et FLOPEC. L'entreprise publique PetroEcuador, la plus importante, administre trois infrastructures majeures : la principale raffinerie du pays, qui débute ses activités en 1978 ; le terminal maritime pétrolier de Balao, seul point d'exportation du pétrole brut national, est situé à $20 \mathrm{~km}$ à l'ouest de l'agglomération d'Esmeraldas; le SOTE (Sistema de Oleoducto TransEcuatoriano), système de conduites qui relie les champs pétrolifères de l'Amazonie à la côte Pacifique, inauguré en 1972. L'entreprise privée OCP (Oleoducto de CrudosPesados S.A.) gère de nouvelles conduites pour l'exportation et l'importation des hydrocarbures (avec son propre terminal maritime), sous la forme d'une concession de 20 ans attribuée à l'inauguration de l'installation, en 2003. Enfin, l'entreprise publique FLOPEC (Flota Petrolera Ecuatoriana $E . P$.), gère la flotte équatorienne qui effectue le transport d'hydrocarbures à partir des terminaux pétroliers situés en mer. Depuis 2015, suite à une décision de l'exécutif national le siège social de FLOPEC est installé sur le Balneario de Las Palmas, en front de mer, au nord de l'agglomération d'Esmeraldas. Le bâtiment lui-même, monumental, fait ressortir la puissance de l'entreprise et de l'État équatorien (fig. 1).

Figure 1 - Le siège de FLOPEC, quartier Las Palmas, sur le front de mer à Esmeraldas 


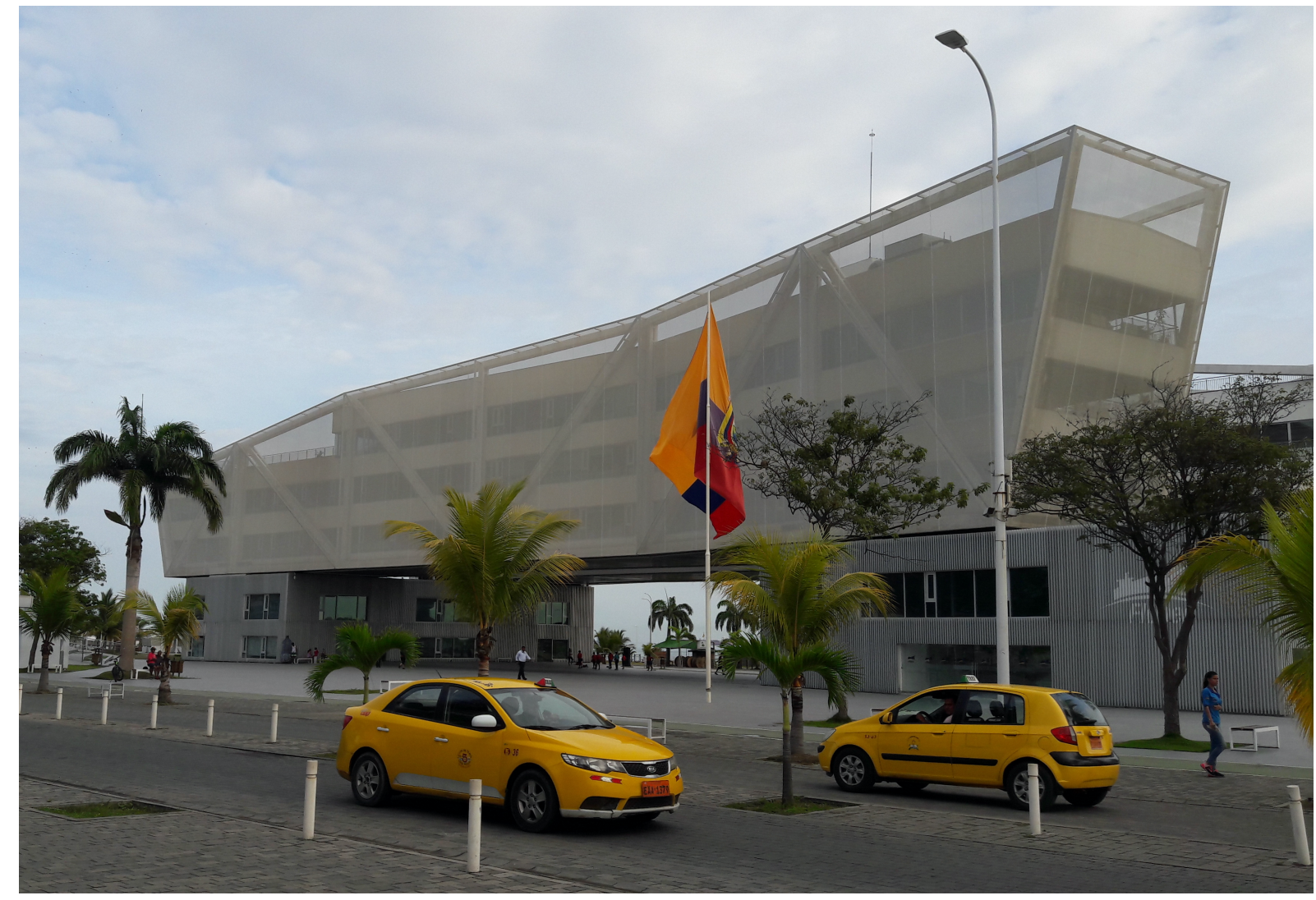

Source : auteurs

A ces principaux acteurs du secteur pétrolier s'ajoute la centrale thermoélectrique de 1'entreprise publique CELEC (Corporación Eléctrica del Ecuador E.P.) inaugurée en 1982 aux portes de la raffinerie, qui fonctionne en lien étroit avec PetroEcuador pour la production d'énergie (fig. 2).

Figure 2 - Distribution des installations pétrolières et des principaux acteurs sur le territoire d'Esmeraldas 


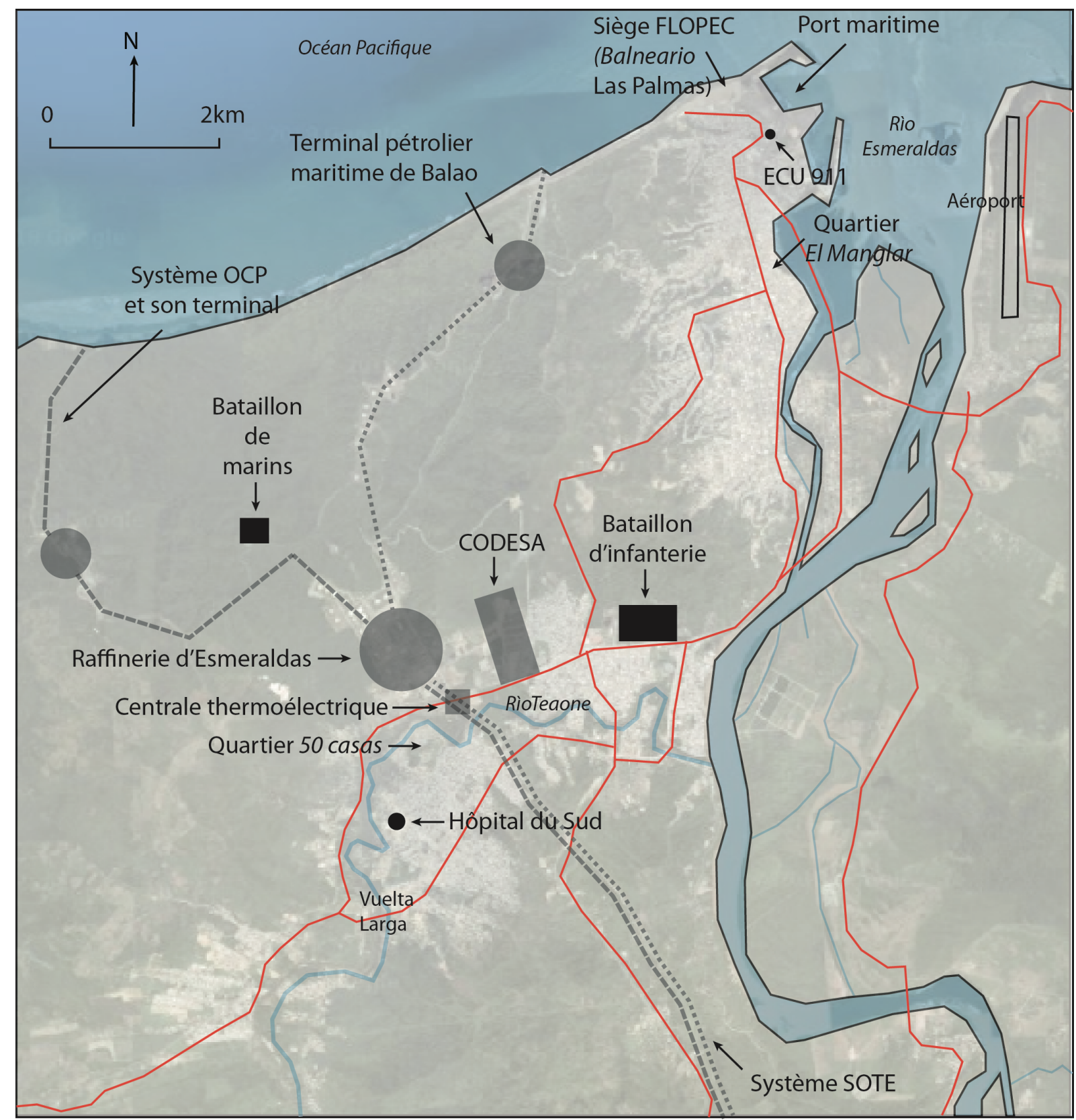

Source. Background map: https://www.google.com/maps/

Source : auteurs

\section{2 Inconvénients et redistribution locale de la ressource en question}

Le caractère stratégique comme les enjeux de souveraineté pour l'Équateur sont vérifiés par les statuts évolutifs des différentes entreprises, oscillant entre contrôle étatique et partenariats privés. La question du contrôle national des ressources et des entreprises liées au secteur pétrolier recoupe celle concernant le partage des bénéfices provenant de la rente pétrolière. Deux lois imposant un rôle social aux entreprises engagées dans le secteur de l'énergie par la distribution de « compensations » ont marqué le début de la décennie 2010 (celles du 10 juillet 2010 et du 19 juillet 2012). 
Les compensations correspondent à la redistribution locale d'une partie de la rente pétrolière. Question éminemment politique. Le cas d'Esmeraldas n'y déroge pas. On en retrouve la trace en consultant des archives de journaux nationaux et locaux, comme El Comercio ou El Telégrafo à Quito ou encore dans La Hora à Esmeraldas. Par exemple, l'article de El Telégrafo du 25 mars 2017 se réfère à la loi de 2010 qui repose le principe des transferts des bénéfices dégagés par les activités liées au secteur pétrolier vers les populations qui ont été impactées par ces activités : «[...]toutes les redevances tirées de l'exploitation des ressources naturelles doivent bénéficier de façon prioritaire et directe aux habitants des secteurs concernés par l'extraction et l'exploitation des ressources d'hydrocarbures ou minières $»^{3}$. Un autre article, dans El Comercio (30 juin 2012), fait ressortir un bilan très négatif de la multiplication des infrastructures liées aux hydrocarbures à Esmeraldas. Une photographie montre le contraste entre la raffinerie et des quartiers à équipement limité, comme celui de La Florida ou des 50 maisons. Le bilan est présenté de manière très abrupte : « le pétrole a entraîné une croissance de la ville, mais n'a pas amené le progrès ». Il mobilise des témoignages de riverains : « Des gens [...] du quartier La Florida, près de la raffinerie, pensent que ces 40 années n'ont pas apporté de développement». [Un témoin] a « vécu 20 ans à proximité de la raffinerie, sans disposer des services de base ». Cet article fait ressortir le problème que pose la coexistence de la ville et des installations pétrolières. En effet, ces infrastructures ont contribué à recomposer la ville, si ce n'est à l'éclater, et cette évolution est dénoncée comme problématique, tant par la presse que par la municipalité d'Esmeraldas. La redistribution locale d'une partie plus importante des ressources liées à la rente pétrolière, nommée compensation, apparaît justifiée. L'article se termine ainsi : " [les personnes interrogées] pensent qu'avec l'exportation du pétrole, c'est la bourgeoisie de la capitale qui s'est enrichie, et non les habitants de la ville».

\subsection{Implications socio-spatiales de l'exploitation de la ressource : transformations urbaines}

Les transformations, voire l'éclatement de la ville d'Esmeraldas, peuvent être aisément reconstituées. Trois grandes logiques se distinguent, transformant la ville en aire urbaine sans qu'y soient développés les services associés, et ce, sous l'influence privilégiée des entreprises du secteur pétrolier (fig. 3).

Figure 3 - Éclatement de l'aire urbaine d'Esmeraldas en 3 grandes zones

${ }^{3}$ Les citations tirées de la presse sont traduites par les auteurs 

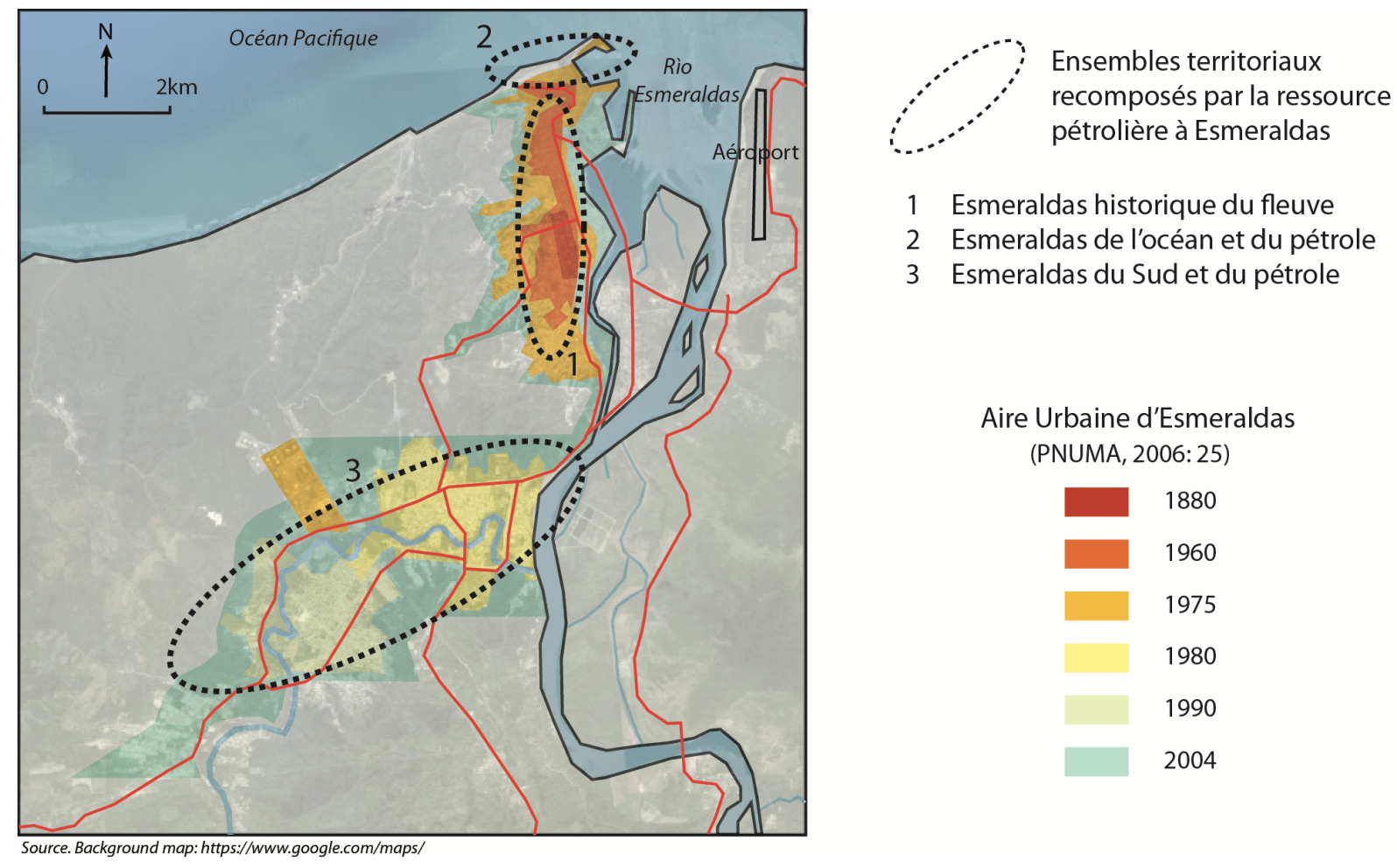

Source : auteurs

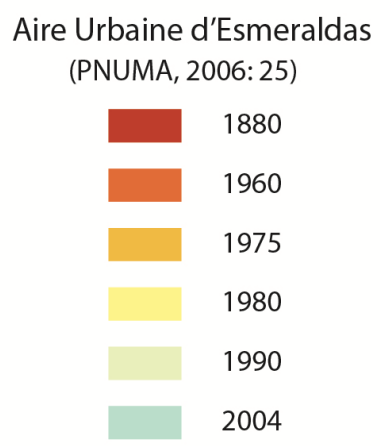

\section{Esmeraldas, la ville et le fleuve}

La ville d'Esmeraldas est historiquement liée au fleuve éponyme. Fondée sur le site de San Mateo, au sud, là où se trouve l'entreprise qui gère l'approvisionnement en eau potable, la ville a été déplacée vers le nord et l'estuaire du río. C'est le long du malecón Maldonado, boulevard anciennement en front de fleuve, que l'on trouve les symboles urbains et officiels principaux : la mairie et le bâtiment qui abrite le siège de la province. Ce dernier est resté l'édifice le plus haut depuis 1984 pour cette partie centrale de l'agglomération. Malgré une urbanisation qui reste morphologiquement limitée depuis la décennie 1980, ce qui reste du passé d'Esmeraldas est peu visible. Le site de San Mateo est délaissé, les édifices associés aux activités de négoce $\mathrm{du} 20^{\mathrm{ème}}$ siècle sont très peu entretenus malgré des volontés récurrentes de mise en valeur (Castillo, 2011, pp. 8-11). Le bâtiment dit La Barraca est emblématique de l'histoire d'Esmeraldas pour plusieurs sources, mais il est très dégradé, et d'aspect quelconque (fig. 4).

Figure 4 - Entrée du centre commercial La Barraca aujourd'hui 


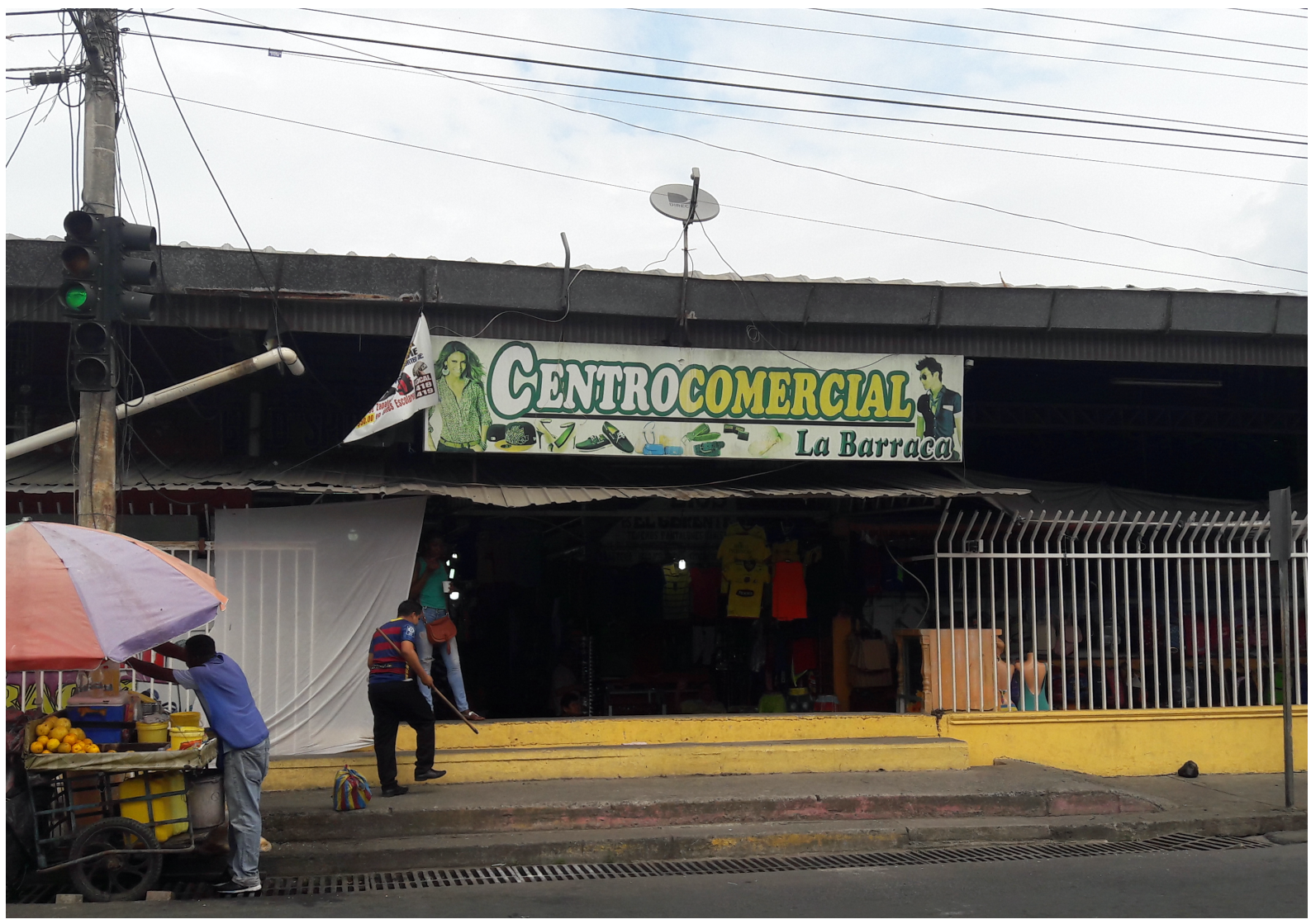

Source : auteurs

C'est également ce que souligne un article de El Comercio daté du 8 août 2010: "Le développement d'Esmeraldas au point mort ». Pour l'illustrer, l'article utilise la photo d'un bâtiment associé au négoce (fig. 5), face au site de La Barraca, qui lui-même prit la place d'un parc associé à la mémoire du héros local (et du personnage national) Luis Vargas Torres.

Figure 5 - Face à La Barraca un bâtiment ancien utilisé pour le négoce très dégradé, que mentionne Castillo (2011) 


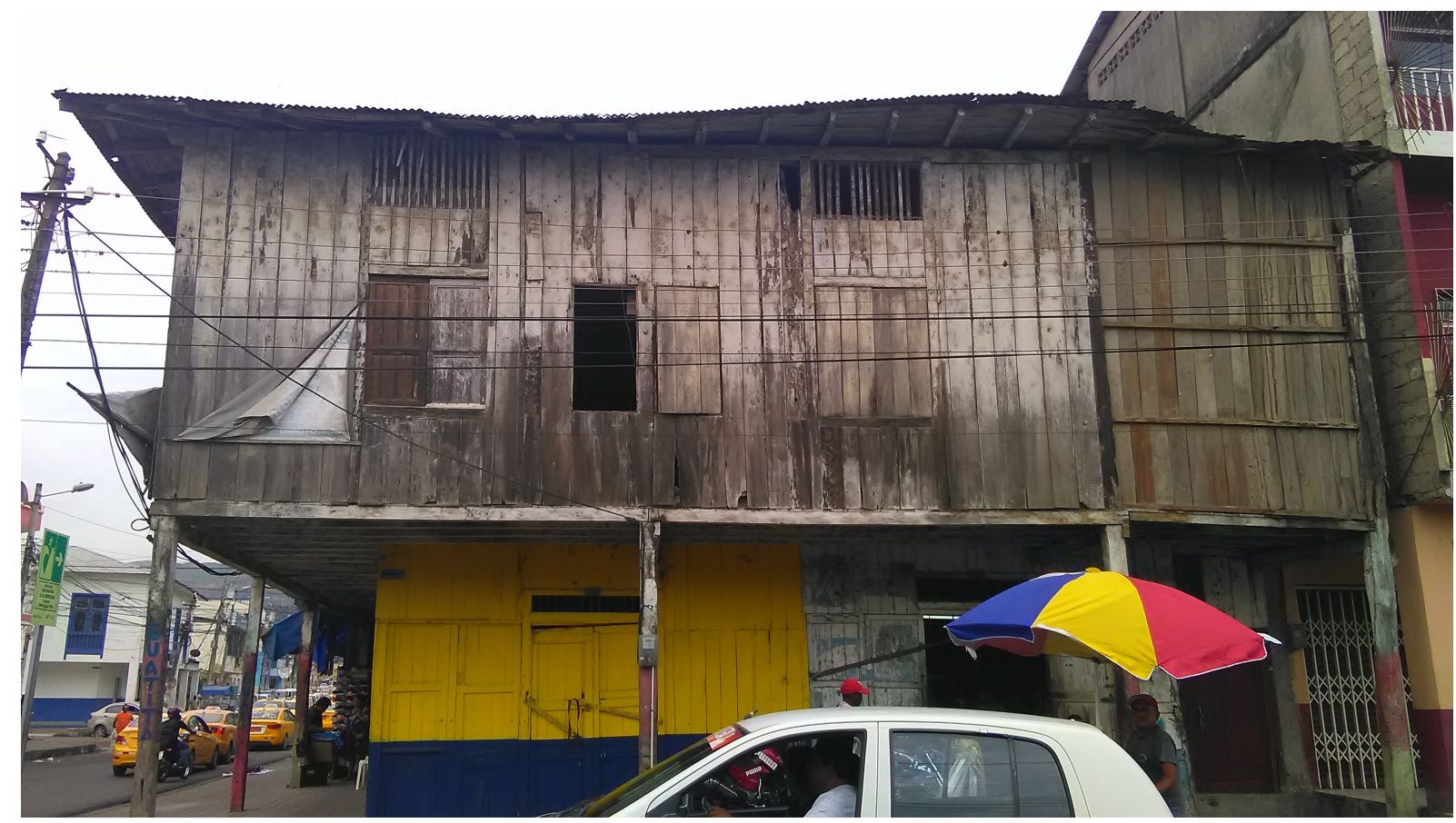

Source : auteurs

Malgré la présence de centralités historiques, Esmeraldas semble, pour ces parties de la ville, oubliée des investisseurs qu'ils soient privés ou publics : pas d'opération de front d'eau, pas de gentrification visible, aucune construction récente d'importance. Les références aux modèles dominants d'aménagement urbain autour de la patrimonialisation de ces traces du passé existent (Castillo, 2011 ; GADME, 2015), et la question fut abordée lors d'un entretien avec un ancien maire d'Esmeraldas, Ernesto Estupiñán. Le déclin du centre fluvial et historique d'Esmeraldas peut sembler étonnant pour une aire urbaine qui rassemble autant d'entreprises puissantes et lucratives. Ce bilan contraste avec l'évolution des deux autres grandes zones toutes deux très fortement - quoique différemment - associées aux entreprises et aux infrastructures liées au secteur pétrolier.

\section{Esmeraldas, l'océan et le pétrole}

Au Nord d'Esmeraldas se trouve le quartier du Balneario de Las Palmas, qui a beaucoup évolué au cours de la décennie 2010. Décrit comme un quartier dangereux et sans réel service (Estupiñán, 1975, p. 24), la seule plage d'Esmeraldas ne comptait qu'une toilette publique, souvent fermée, ce qui obligeait les usagers « à faire leurs besoins dans le sable » (La Hora du 7 février 2007), et accueillait des clubs nocturnes et des lupanars jusqu'à la fin des années 2000. Le Balneario de Las Palmas est aujourd'hui devenu la vitrine de l'agglomération. Le symbole de cette évolution est l'installation du siège de FLOPEC. Il contraste très fortement avec les bâtiments officiels municipaux ou provinciaux du centre historique d'Esmeraldas. Dans le quartier de Las Palmas, c'est l'État équatorien qui, par l'intermédiaire de FLOPEC et sur la base du principe de compensation, impose une évolution de l'urbanisation qui est supposée 
favoriser le tourisme, et donner à Esmeraldas un front de mer moderne et attractif qu'elle n'avait pas jusqu'alors. Toutefois, l'investissement de plus de 50 millions de dollars nécessaire à la réhabilitation du front de mer peine à « entraîner » le reste du territoire d'Esmeraldas.

\section{Esmeraldas, « le Sud » et le pétrole}

À l'opposé, au sud-ouest d'Esmeraldas, de nouveaux quartiers ont progressivement rejoint une partie des infrastructures liées au secteur pétrolier. Ces dernières ont été initialement localisées à l'écart de la ville d'Esmeraldas. Leur accès demeure aujourd'hui très limité, et en partie contrôlé par l'armée. Un bataillon de la Marine est localisé à la jonction des deux routes qui desservent les infrastructures OCP et SOTE à proximité du littoral et du terminal maritime de Balao. Pour autant, les projets d'aménagement du sud de la ville portés par la mairie et la province, et présentés par le Bureau Intégré de Planification d'Esmeraldas dans les années 1970 (Oficina Integrada de Planificación de Esmeraldas- OIPE, 1975), ne faisaient pas une obligation de la mise à distance entre l'urbanisation et les installations pétrolières. Le rapport prévoyait de développer des services dans les quartiers du sud, comme l'approvisionnement en eau potable, l'électricité ou le réseau d'égouts, accompagnant la trame viaire. Malgré le développement de l'urbanisation, ces services n'existent aujourd'hui qu'en partie.

Le rapport OIPE envisageait plusieurs scénarios. Certains définissent des zones tampons de 20 mètres au moins entre les quartiers planifiés et les infrastructures, ou même par rapport au río Teaone (OIPE, 1975, p. 106). Aujourd'hui, le contact entre certaines de ces infrastructures et les quartiers habités est quasiment direct, comme cela se vérifie avec le quartier dit des 50 maisons. Il est seulement séparé de la centrale thermoélectrique par le rio Teaone (fig. 6). Le développement du bâti vers le sud-ouest et les infrastructures liées au secteur pétrolier s'est produit essentiellement par construction spontanée. Il n'a pas été accompagné des services prévus initialement malgré une importante croissance des populations pauvres attirées par les promesses de développement liées à l'activité pétrolière (CONADE, 1980).

Figure 6 - Quartier 50 casas, séparé de la centrale thermoélectrique par le río Teaone 


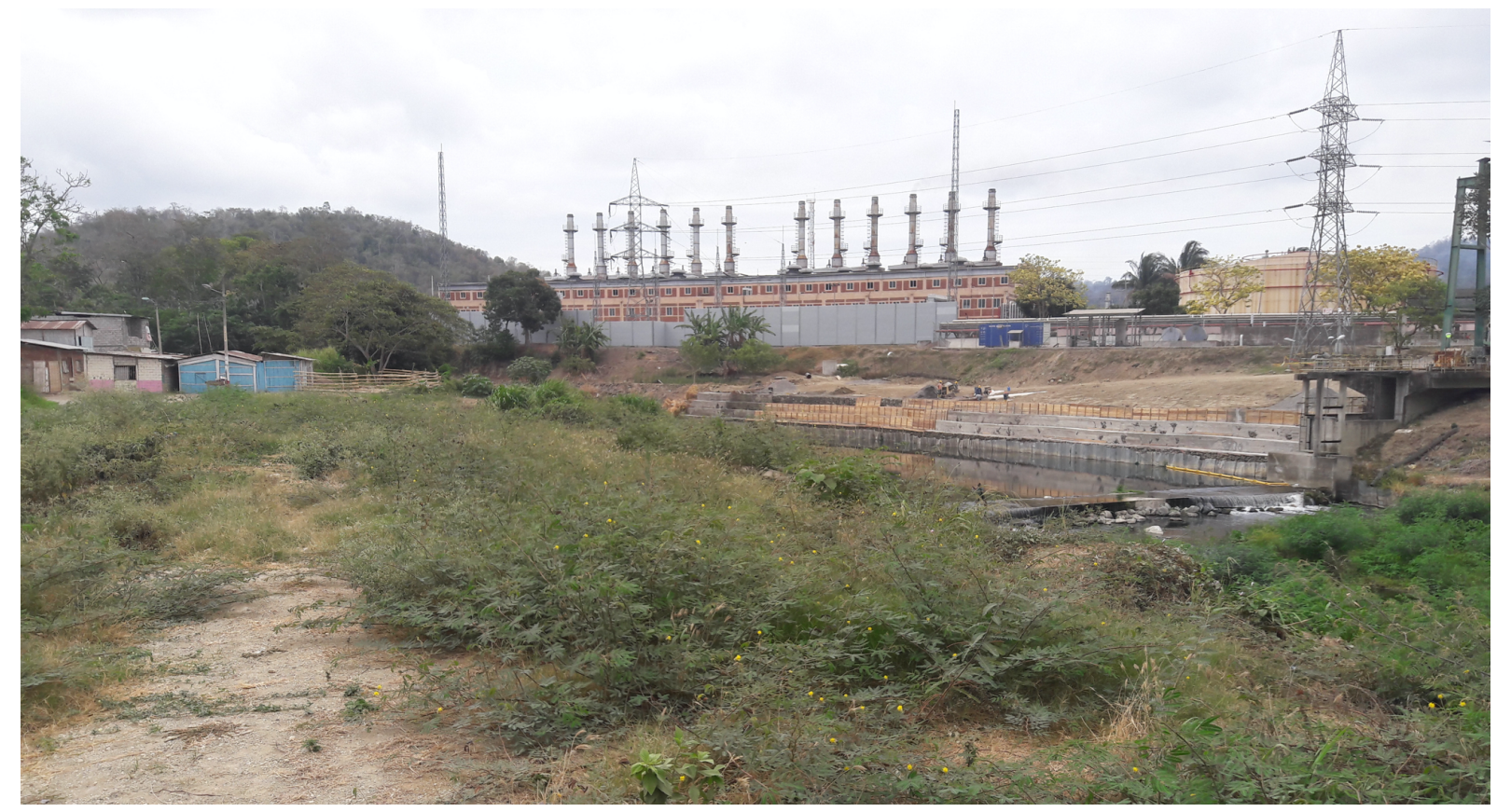

Source : auteurs

Cette situation correspond au moins en partie à celle qui est dénoncée par les articles des journaux précités, ou lors des entretiens avec différents acteurs locaux : si la rente pétrolière n'a pas amené les équipements et services nécessaires à l'urbanisation, elle a cependant transformé la ville d'Esmeraldas. La réhabilitation du Balneario de las Palmas et l'installation du siège de FLOPEC dessinent un projet urbain que les élus locaux ne peuvent ni financer ni contrôler.

\section{La ressource pétrole modifie et multiplie les risques}

Les activités pétrolières ont non seulement reconfiguré l'urbanisation d'Esmeraldas, mais également le panorama des risques sur ce territoire, tant à l'échelle locale qu'à l'échelle nationale, d'une part en positionnant des enjeux majeurs nationaux sur un territoire très vulnérable, d'autre part en introduisant de nouveaux types de risques à l'échelle locale. Ce processus a ainsi augmenté la vulnérabilité à la fois locale et nationale, tout en multipliant les interactions possibles entre aléas. Il transforme les modalités de mise en œuvre des politiques de gestion des risques par l'éclatement des acteurs et des objectifs qu'ils poursuivent et produit une fragmentation des territoires et la transformation des risques. Par exemple, la construction de la centrale thermoélectrique et l'édification du quartier dit des 50 maisons le long des berges $\mathrm{du}$ Rio Teaone modifient les risques dits technologiques, pour l'entreprise et pour les populations riveraines, mais aussi les risques d'inondations. 


\section{1 De l'approche centrée sur l'aléa au système multi-risques}

En se limitant dans un premier temps à la vision classique des risques centrée sur les aléas, le secteur d'Esmeraldas se distingue par une forte exposition à d'importants aléas d'origine naturelle, rendant a priori l'endroit peu propice à l'installation d'infrastructures stratégiques.

\section{De multiples aléas}

Le risque sismique et de tsunami est particulièrement élevé à Esmeraldas. En effet, la ville est située sur la côte Pacifique, à proximité d'une marge continentale active et les sismologues estiment que la probabilité d'un séisme majeur dans ce secteur est particulièrement élevée. Les données de l'institut géophysique de Quito font état de 5 séismes de magnitude égale ou supérieure à 7 ayant touché Esmeraldas depuis le début du $20^{\text {ème }}$ siècle, en 1906, 1942, 1958, 1979 et 2016. Les informations relatives à la période antérieure sont lacunaires voire contradictoires (Rebotier et al., 2019). Le séisme de 1906, d'une magnitude 8.8 dont l'intensité a été estimée à IX sur l'échelle de Mercalli (Serrano et D'Ercole, 2003), est considéré comme l'un des plus importants enregistrés dans le monde. Accompagné d'un tsunami, il a fait des dizaines de morts et des dommages considérables. Un autre tsunami a aussi marqué les esprits en 1958. Le risque volcanique est généralement passé sous silence, notamment en raison de l'éloignement des volcans, la fréquence rare des éruptions, mais aussi de sa relativisation par rapport aux risques de la vie quotidienne de la population. Cependant l'éruption du volcan Cotopaxi en 1877 a engendré des lahars qui sont allés jusqu'au Pacifique, provoquant une forte crue jusqu'à l'embouchure du fleuve Esmeraldas.

La configuration du site, à la fois littoral et fluvial, en fait un secteur soumis de manière récurrente aux inondations dans les parties basses et aux glissements de terrains dans les secteurs de collines (fig. 7). En période de Niño, ces phénomènes sont particulièrement intenses. Ainsi, en mars 1976, des pluies intenses ont été enregistrées entrainant un glissement de terrain qui a provoqué la mort de 60 personnes. En 1983, plus de 1000 logements ont été détruits. En 1993, un glissement de terrain a laissé un solde de 83 maisons détruites. En $1997-$ 1998 un épisode de Niño particulièrement virulent a provoqué de fortes crues et des coulées de boues (Perrin et al., 1998). Au-delà de ces évènements, les inondations affectent très régulièrement la partie du territoire anciennement urbanisée, touchant des populations très pauvres situées en bord de fleuve dans des zones de mangroves plus ou moins stabilisées sur les rives du río Esmeraldas, comme cela a été le cas en janvier 2016.

Figure 7 - Une ville exposée aux glissements de terrains et aux inondations 


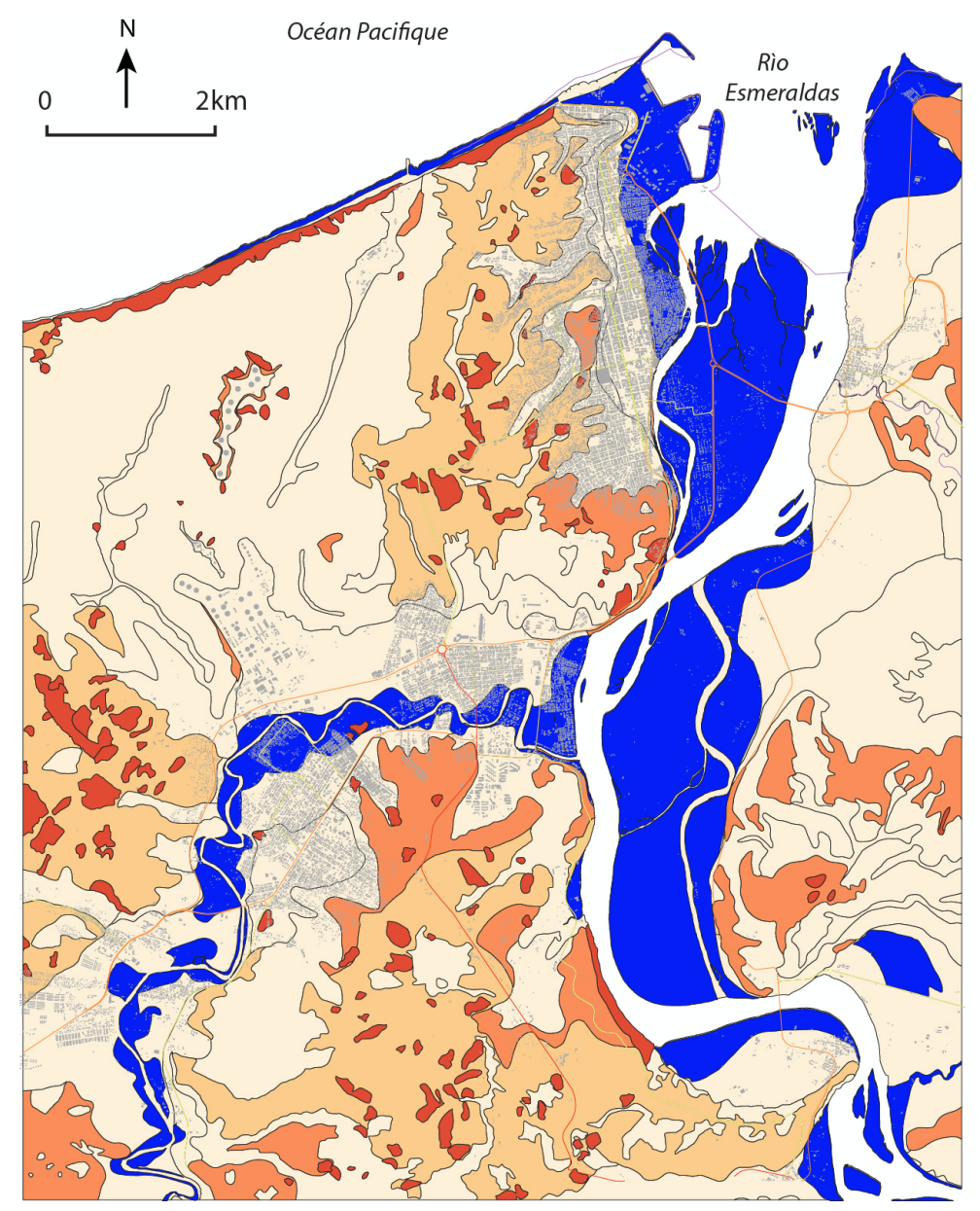

Secteurs inondables

Exposition aux glissements de terrain

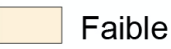

Moyenne

Importante

Très importante

Source: d'après le Gobierno AutónomoDescentralizado del Municipio de Esmeraldas (GADME)

Les catastrophes qui touchent Esmeraldas sont donc le résultat de son occupation urbaine combinée à des conditions physiques du site particulièrement défavorables. La modification du site, notamment le remblaiement des quebradas, l'absence ou l'insuffisance du réseau d'évacuation des eaux pluviales et la déforestation, mais aussi l'urbanisation de pentes instables ou l'occupation des berges du fleuve ont provoqué ou amplifié les phénomènes physiques destructeurs (fig. 8).

Figure 8 - Quartier El Manglar, exposé aux inondations sur des terrains gagnés à la mangrove 


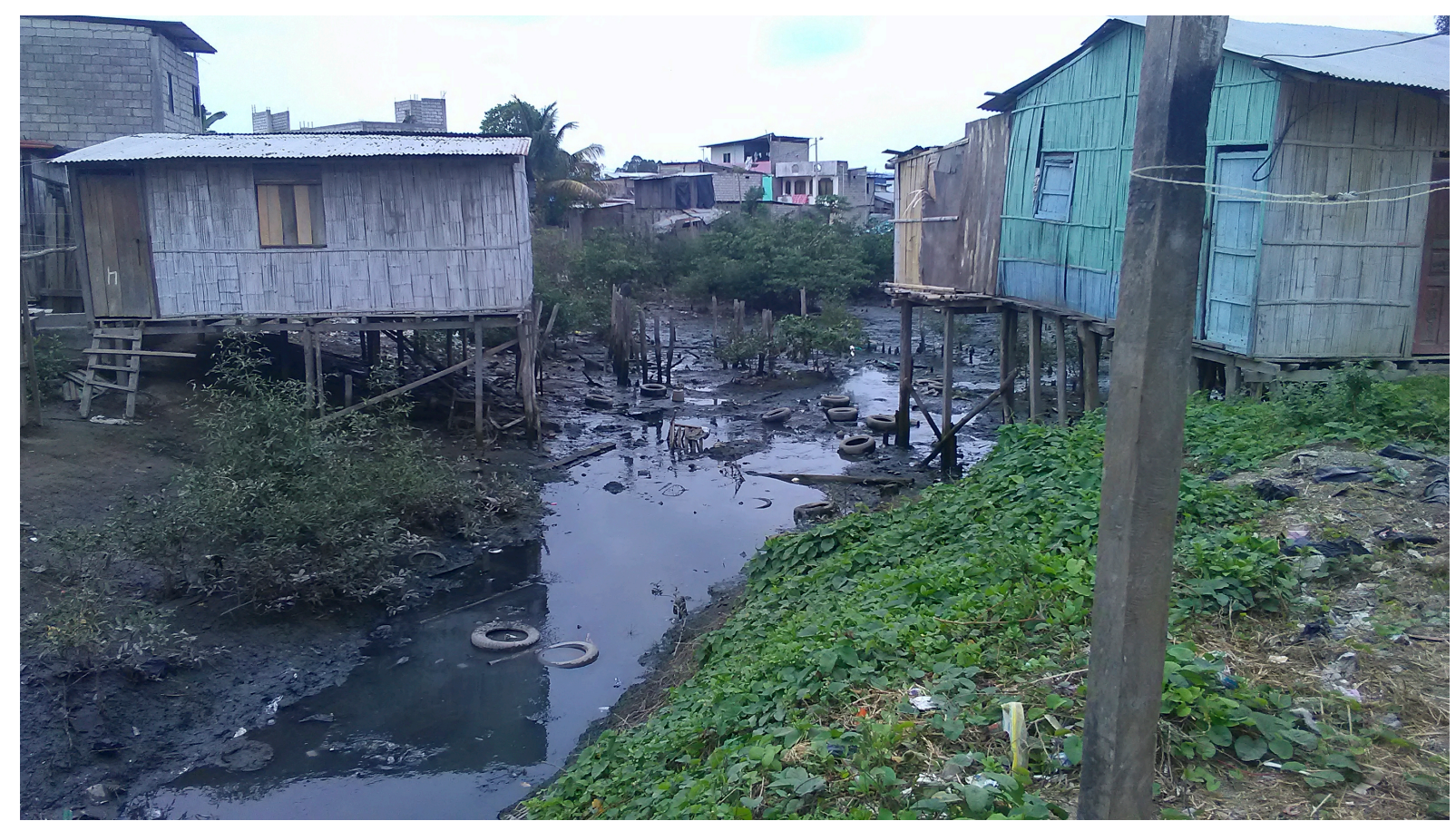

Source : auteurs

\section{L'installation des activités pétrolières, produit d'un arbitrage entre différents types de risque}

Même si dans les années 1970 la problématique des risques n'avait pas l'importance qu'on lui accorde aujourd'hui, on ne pouvait ignorer que Esmeraldas était une zone très exposée, à la fois aux séismes, aux tsunamis, aux inondations et aux glissements de terrain. Il est clair que le choix du site d'Esmeraldas pour l'implantation des infrastructures pétrolières d'exportation et de raffinage répond à des logiques nationales, politiques et géopolitiques, qui ont primé sur la prise en compte de la situation locale, du contexte social et des risques dits naturels.

La nécessité était alors de transporter le pétrole depuis les zones d'exploitation de l'Amazonie vers un port sur le Pacifique. Le choix d'Esmeraldas peut s'appuyer en premier lieu sur l'argument technique et économique de la proximité (relative) des champs pétrolifères en exploitation dans l'oriente (Shushufindi, province de Sucumbíos), réduisant d'autant la longueur de l'oléoduc de pétrole lourd à construire (le SOTE). Par ailleurs, on retrouve l'argument classique d'utiliser l'activité pétrolière pour contribuer au développement régional de la province d'Esmeraldas, l'une des plus pauvres de l'Équateur (OIPE, 1975).

Cependant, d'autres arguments peuvent être avancés, à la fois politiques et géopolitiques. D'une part, la rivalité historique entre Quito, capitale de l'Équateur et Guayaquil, premier port et capitale économique du pays, a joué un rôle dans ce choix. Dans cette compétition, la dynamique économique attendue de l'exploitation pétrolière devait apporter un atout à Quito (Dalmasso et Fillon, 1970). Aussi, l'exportation du pétrole, principale ressource économique du pays, ne devait pas être laissée à Guayaquil qui joue déjà un rôle économique majeur. 
L'exportation du pétrole via Esmeraldas a donc permis de limiter l'importance de Guayaquil en positionnant les activités de transformation et d'exportation pétrolière dans la sphère d'influence de Quito (Collin Delavaud, 1978).

Par ailleurs, dans les années 1970, les relations conflictuelles avec le Pérou fournissent un argument supplémentaire autour de la sécurité nationale au gouvernement militaire de l'époque, pour situer le port exportateur de pétrole et la principale raffinerie du pays loin au nord, puisque le voisin du sud est historiquement considéré comme un agresseur. Autrement dit, il fallait implanter les infrastructures stratégiques hors de portée des forces militaires péruviennes, bien au nord de Guayaquil.

Sans avoir accès aux documents du processus de décision qui a conduit au choix d'Esmeraldas, mais étant donné la forte vulnérabilité due à son exposition à de multiples aléas soulignés précédemment, on peut considérer que des enjeux nationaux et des risques politiques de contrôle territorial et géopolitiques de souveraineté et de sécurité nationale ont largement contribué au choix d'Esmeraldas. Ces éléments confirment le fait que gérer un risque c'est faire un choix dans un système de risque (Dourlens et al., 1991). Les arbitrages qui s'opèrent se font alors en fonction des échelles, des enjeux et des acteurs, et en l'occurrence, l'acteur local n'a que très peu de poids.

\subsection{Augmentation et complexification des risques à l'échelle locale}

Les infrastructures pétrolières qui sont situées sur le territoire d'Esmeraldas concernent à la fois des opérations de transport, de stockage, et de transformation, situées sur terre et en mer. Qu'il s'agisse de la raffinerie, des oléoducs, du terminal maritime, des réservoirs, des bouées de ravitaillement en mer des pétroliers, chacune de ces infrastructures représente un réel danger. La centrale thermoélectrique située à proximité immédiate de ces installations pour bénéficier de l'apport de combustible qui lui est nécessaire contribue elle aussi à la production des risques (fig. 2 et 6).

Si le site autour de ces installations était pratiquement vide de population à l'époque de sa construction au début des années 1970, il est aujourd'hui à proximité immédiate des quartiers populaires, suivant la dynamique urbaine impulsée par les activités pétrolières. Ceux-ci se sont développés au sud de l'agglomération le long de la route à Atacames (secteur Vuelta Largafig. 3) et se trouvent de ce fait dans la zone qui serait directement impactée par un accident technologique majeur.

Toutes ces installations sont dangereuses, à la fois pour la population, le territoire situé à proximité, et le milieu environnant. Les accidents les plus courants sont les incendies, les explosions, les fuites de gaz et de carburants qui sont la matérialisation brutale des risques technologiques. Esmeraldas a connu quantité de fuites et déversements accidentels de pétrole, dont la littérature déjà citée (grise comme académique) rend partiellement compte. 
En parallèle, les activités pétrolières produisent ce que l'on peut appeler des risques lents par contamination de l'eau, de l'air et des sols, qui à leur tour engendrent des risques sanitaires importants pour la population. Des mesures effectuées récemment (Harari et al., 2016; Maurice, 2018 ) font la démonstration que la pollution de l'air à Esmeraldas est très importante, ce qui ne peut manquer d'avoir un impact direct sur la santé des habitants.

Au-delà de ces nouveaux risques, l'enchainement entre les différents types de risques a été démontré en février 1998, quand le fleuve Esmeraldas a pris feu. Le déroulement des évènements était totalement prévisible. Une période de Niño particulièrement forte se traduisit par des pluies intenses qui provoquèrent un glissement de terrain. Ce dernier entraîna la rupture de l'oléoduc qui conduisit à un déversement de pétrole qui s'embrasa dans un incendie. Une boule de feu s'écoula dans la rivière Teaone puis dans le fleuve Esmeraldas et aboutit au décès d'une vingtaine d'habitants et à la destruction d'un quartier. De la même façon, un séisme peut provoquer la rupture d'une conduite de pétrole et impacter directement non seulement l'environnement et la santé des habitants, mais toute l'économie du pays comme le séisme amazonien de 1987.

Esmeraldas présente donc aujourd'hui un panorama de risques multiples et susceptibles d'entrer en interaction et de se renforcer mutuellement (sécurité industrielle, risques environnementaux, risques sanitaires, risques technologiques, risques d'origine naturelle). Un tel constat rend d'autant plus surprenant l'éclatement entre les logiques et les acteurs de leur gestion, qui accroît les difficultés à réduire les risques sur le territoire malgré l'importance stratégique de la ressource pétrolière.

\section{La ressource pétrole fragmente la gestion du risque et du territoire}

Les processus de transformation, fragmentation et différenciation des différents types de risques et des dynamiques territoriales par les activités pétrolières ont des conséquences sur la gouvernance d'Esmeraldas. Le découplage entre risques, ressource et territoire constitue un obstacle important à la réduction des risques de désastres, et contribue même à les augmenter.

\section{1 Des difficultés accrues par une gouvernance des risques fragmentée}

\section{Le gouvernement municipal d'Esmeraldas (GADME), acteur clé et fragile de la gestion des risques}

Depuis la Constitution de 2008, le gouvernement municipal occupe un rôle pivot dans l'organisation règlementaire du système national de gestion des risques en Équateur. Deux

\footnotetext{
${ }^{4}$ http://www.monoil.ird.fr/
} 
explications principales permettent cependant de comprendre la fragilité de ce niveau de pouvoir pour gérer les risques à Esmeraldas.

Pour une part, les capacités à la fois techniques, humaines et financières du GADME sont particulièrement faibles, ce qui limite drastiquement l'impact des politiques locales en général et de gestion des risques en particulier. On trouve là des traits communs à la plupart des gouvernements locaux en Équateur (Rebotier, 2016). Les outils et règlementations de la planification peinent à se concrétiser sur le terrain et la prise en compte des risques n'est pas du tout homogène d'un GAD municipal à un autre. A Esmeraldas, le travail de terrain ne nous a pas permis d'identifier les documents de référence pour la règlementation de l'usage du sol, ou encore le cadastre.

Ainsi, malgré l'existence de quantité d'informations, d'études et de rapports, réalisés localement à Esmeraldas, avec l'aide d'institutions nationales et internationales, ou encore avec l'aide de la coopération, on ne trouve guère de traduction opérationnelle de ces documents techniques. Le PDOT évoque les risques de façon sélective (centrés sur les aléas d'origine naturelle - inondations, glissements de terrain) et en toute généralité, sans transcription aisée vers la planification ni l'usage du sol (GADME, 2012 ; 2015). Le document stratégique de Gestion des risques du canton Esmeraldas (GADME-OXFAM, 2012) ne fait pas mention des problèmes liés aux installations pétrolières, alors même qu'il souligne la priorité qui doit être donnée à la gestion intégrée des risques sur le territoire. La gestion de ces problèmes est abandonnée aux acteurs du secteur pétrolier. En outre, la seule pollution dont il est question provient de la circulation automobile et des déchets domestiques. Les risques technologiques directs liés à la raffinerie ou à la centrale thermoélectrique ne sont pas mentionnés.

Les informations et documents relatifs à la gestion des risques apparaissent limités, dispersés, hétérogènes, et peu utilisables concrètement.

D'autre part, la primauté d'une ressource stratégique gérée de façon centralisée s'accompagne d'un contrôle important de l'emprise territoriale correspondante. Les parcelles de la raffinerie, des oléoducs, des terminaux pétroliers, ou encore des installations annexes nécessaires au fonctionnement du secteur (piscines, réservoirs, etc.) échappent à l'autorité municipale. Si on ajoute aux emprises territoriales du secteur pétrolier celles d'autres grands acteurs nationaux, comme l'armée (avec le bataillon d'infanterie, le corps d'ingénieurs, La Marine), ou encore le port commercial, sous la tutelle du ministère des transports et travaux publics (fig. 2), l'espace juridiquement placé sous l'autorité municipale s'en trouve réduit d'autant.

\section{Le secteur pétrolier, des acteurs puissants pour une approche sectorielle des risques}

Le secteur pétrolier affiche quant à lui une logique sectorielle prioritaire de continuité du service, de protection des installations et de sécurité industrielle. La conception du risque dans le milieu pétrolier est bien illustrée par l'entreprise OCP qui évalue le risque pour son personnel, le risque économique, le risque opérationnel de suspension de ses activités, le risque de perdre 
des clients. Pour ce qui concerne l'impact territorial, les risques qui sont envisagés sont principalement le risque environnemental, l'indignation de la population et les dommages à son image. A aucun moment il ne semble y avoir de collaboration avec le GADME pour la mise en place d'une politique de réduction des risques qui prenne en considération l'impact d'un désastre lié au pétrole sur le territoire.

En outre, les capacités techniques et financières (mais aussi politiques) de ces acteurs du pétrole s'avèrent sans commune mesure avec celles que peut déployer le GADME. La gouvernance du secteur est pour beaucoup assurée depuis Quito, par l'administration des entreprises publiques et le ministère des hydrocarbures. La décision de l'exécutif national de transférer le site de FLOPEC à Esmeraldas prend pour motif la correction d'asymétries de développement, et un certain rééquilibrage territorial. Mais nombre de fonctionnaires à responsabilité sont toujours originaires de la Sierra, et non de la côte : l'effet d'entraînement sur le développement local peine à se concrétiser y compris dans le recrutement des fonctionnaires. Enfin, la fiscalité des activités pétrolières lucratives échappe pour beaucoup aux caisses municipales, alors que les initiatives de compensation, conséquentes mais fragmentées et non coordonnées, ne pallient que très symboliquement le déficit flagrant d'équipements et de capacités institutionnelles locales. Ainsi entre 2000 et 2018, PetroEcuador a investi près de 160 millions de dollars dans la seule agglomération d'Esmeraldas au titre des activités de « relations communautaires », soit près de 8,5 millions de dollars par an, une part considérable du budget global du GADME qui avoisinait les 30 millions de dollars en 2017. L'obligation de cette redistribution, qui peut se concrétiser de façons très différentes, s'applique à l'ensemble des acteurs du secteur pétrolier qui intervient sur le territoire d'Esmeraldas. Or si ces compensations répondent à des obligations légales, leurs critères de distribution ne sont pas d'une grande clarté. Début 2019, des règles d'attribution de ces dépenses (localisation, domaine, montant, etc.) étaient en cours de formulation au sein du département des relations communautaires de PetroEcuador.

Dans un état de grand dénuement, difficile pour le GADME de s'opposer à la construction d'un équipement supplémentaire en ville, même si l'emplacement n'est pas cohérent avec l'usage du sol du quartier (ou avec les programmations ministérielles, pour l'éducation ou la santé), se situe dans une zone inondable, ou à l'intérieur du périmètre de danger de l'explosion potentielle des installations de la raffinerie. D'ailleurs, PetroEcuador est à l'initiative de la construction d'une école dans le quartier des 50 maisons qui cumule les inconvénients cités précédemment.

Des investissements conséquents existent sur le territoire d'Esmeraldas. Mais leur localisation augmente les risques et s'inscrit à l'encontre d'une stratégie coordonnée de gestion des risques, en particulier dans les espaces les plus dangereux. Plus que des effets de rattrapage, ou véritablement de compensation, ces dépenses précipitent une plus grande désorganisation de la gouvernance territoriale locale et une augmentation des risques.

La gestion des risques (et du territoire) est éclatée entre plusieurs acteurs qui relèvent d'échelles différentes, qui n'ont pas les mêmes objectifs, les mêmes logiques, les mêmes conceptions des risques, les mêmes capacités et compétences, ce qui les inscrit dans un rapport de force profondément asymétrique. Mais une révision des raisons multiples qui contribuent à expliquer un tel éclatement permet de ne pas faire de la seule présence du pétrole la cause de tous les 
maux. Elle montre également le difficile chemin à parcourir pour œuvrer à plus d'intégration afin de réduire les risques durablement et pour tous.

\subsection{Les multiples ressorts de la fragmentation des risques et de leur gestion}

Le kaléidoscope des risques à Esmeraldas souligne l'importance de conceptions multi-acteurs et multirisques. Mais la possibilité d'une intégration (de conceptions, de connaissances, d'intérêts, de périmètres d'action des acteurs, etc.) est concrètement entravée par de multiples éléments. Nous revenons ici sur un certain nombre de ces éléments, liés pour beaucoup à la gouvernance (des risques comme du territoire) et aux rapports qu'entretiennent les acteurs entre eux.

\section{Entre autonomie et intervention : tensions entre pouvoirs asymétriques}

Les accrocs politico-institutionnels à la décentralisation sont nombreux en Équateur, et la gestion des risques est un des théâtres de ces conflits (Rebotier, 2015).

Même si la municipalité est constitutionnellement autonome dans le domaine de l'aménagement et de la planification sur son territoire, les formes d'interventions directes d'autres acteurs ou échelons de pouvoirs sont très nombreuses, qui associent inégalement la voix du gouvernement local. Dans le cas d'Esmeraldas, le motif d'une intervention directe de l'État est souvent celui de la promotion du développement local et du paiement d'une dette historique liée à l'exploitation de la ressource pétrolière affectant le territoire. Si on a mentionné les ambigüités des dépenses directes des acteurs du secteur pétrolier au titre des «compensations », d'autres initiatives d'État s'imposent, sans qu'on ne comprenne véritablement ni l'articulation au développement local - peu explicite au demeurant - ni la logique de localisation des projets. C'est le cas de l'inauguration en 2017 de l'Hôpital du Sud (dans les quartiers du Sud) pour plus de 80 millions de dollars alors qu'un autre hôpital est abandonné dans le centre (la «ville du fleuve ») ; de la réhabilitation du front de mer (entre 2014 et 2016) pour plus de 50 millions de dollars, pleinement exposé au tsunami; ou de la construction du centre de coordination des secours (ECU 911), dans la zone inondable de l'estuaire du fleuve (fig. 2 et 7).

Si la redistribution locale d'une partie de la rente pétrolière existe bien (par le biais des compensations du secteur pétrolier, ou par celui des investissements d'État), elle échappe majoritairement au contrôle ou à l'arbitrage du GADME. Ce type de transfert s'opère dans le cadre d'une relation asymétrique entre acteurs nationaux (qui disposent de la manne pétrolière) et locaux (légalement autonomes pour les choix d'aménagement sur leur territoire). Cette tension asymétrique entre autonomie et intervention répond tout à la fois à l'obligation légale de transférer la richesse des ressources vers les territoires les plus affectés ou impliqués, à la recherche d'un effet de rattrapage de développement pour une province marginale et jugée « en 
retard ॥; ou localement, à la recherche d'un effet d'entraînement du secteur touristique dans une ville pauvre.

De tels transferts multimillionnaires n'échappent pas à l'instrumentalisation politique. Sous couvert de contourner les pratiques clientélistes, l'incompétence, voire la corruption de la gestion municipale, les acteurs du secteur pétrolier gardent le contrôle des compétences disponibles localement, ainsi que de la richesse pétrolière qui, légalement, doit bénéficier aux territoires affectés. L'État central, quant à lui, compte sur un effet de levier de ces investissements massifs engagés en toute autorité, pour les porter au crédit de sa popularité face à des forces politiques locales adverses. Si le pouvoir municipal a été arraché en 2014 par l'État central à un des secteurs de l'opposition, il est à nouveau devenu en 2019 un bastion du MPD, parti d'opposition.

\section{Le passif tenace d'une histoire politique conflictuelle}

La rivalité politique entre l'État central et le pouvoir local prend un tour singulier à Esmeraldas dont seule l'histoire politique permet de prendre la mesure. Esmeraldas est souvent présentée au titre de spécificités culturelles, voire ethniques (au motif d'une population afro-descendante dominante), qui expliquerait un rapport politique tendu au pouvoir central. Historiquement, et dans l'imaginaire national, Esmeraldas est la province rebelle. Terre renégate, au pouvoir de la colonie comme au conservatisme postérieur à l'indépendance, Esmeraldas est également une terre indomptable de la République. Il s'agit aussi du dernier front pionnier de la région littorale, à la fin du $20^{\text {ème }}$ siècle (Collin Delavaud, 2018), ce qui lui vaut le diagnostic d'un « retard » structurel d'équipement et d'une dette historique au regard de son niveau de développement.

Sans remonter aux épisodes de la conquête ou à l'établissement dans la région d'Esmeraldas d'une République Zamba qui tint tête aux colons espagnols jusqu'au milieu du $16^{\text {ème }}$ siècle (Rueda Novoa, 2001), la permanence d'une telle irréductibilité dans le temps peut donner d'autres clés de compréhension de la conflictualité et de la fragmentation des interventions portées actuellement par de multiples acteurs nationaux sur le territoire municipal.

\section{Des rapports difficiles accentués par la présence de la ressource pétrolière}

Aujourd'hui, les relations entre les acteurs du secteur pétrolier, les acteurs nationaux, et le pouvoir public municipal s'établissent principalement sous le signe de la menace et de la défiance. Après les élections municipales de mars 2019, la nouvelle administration a établi un premier contact avec PetroEcuador, en les informant d'un dépôt de plainte motivé par les dommages causés à la population locale après plus de 40 ans d'exploitation (et de pollution) de la raffinerie. Même si l'on comprend ce premier geste comme une façon musclée pour la maire fraîchement élue d'entrer dans la négociation avec un acteur puissant, le rapport frontal ne laisse pas présager d'articulation aisée entre les approches sectorielles du risque (liées aux activités pétrolières) et celles posées par des questions de développement humain et territorial. On voit 
là se rejouer des tensions entre des conceptions différentes du développement, au sein desquelles environnement et ressources naturelles occupent des statuts différents; mais aussi des rapports conflictuels entre échelons de pouvoir dans un pays engagé dans un processus de décentralisation (Le Quang et Ramirez Gallegos, 2016). Étant donné ces rapports conflictuels, la possibilité d'une conciliation et d'arbitrages entre intérêts multiples (situation multi-acteurs et multirisques) se trouve hypothéquée.

En pratique, depuis la Constitution de 2008, les documents de gestion des risques des entreprises publiques et privées opérant sur le territoire d'une municipalité doivent passer par le contrôle du service municipal dédié. Mais quelle peut être la capacité de validation des quelques agents de l'unité de gestion des risques du GADME lorsqu'ils reçoivent la documentation des plans de contingence de la raffinerie ou du terminal pétrolier ? Du reste, reçoivent-ils toute la documentation ? Que peuvent-ils exiger ? Suggérer ? Demander, au titre des impacts constatés ou supposés sur le territoire?

Dans ces interactions délétères de dépendance et de conflictualité, les pouvoirs publics locaux ne sont pas seulement les victimes d'une décentralisation biaisée, ou dépossédés par le surinvestissement de puissants acteurs extérieurs. Ces tractations sur le territoire, entre risques et ressources, font aussi l'objet de marchandages, de compromis, d'effets d'opportunité. Le manque de transparence dans les procédures, l'absence de documents de référence et le profond fossé qui existent entre des acteurs aux pouvoirs, compétences et capacités si différents accroissent l'opacité de la gouvernance et son caractère discrétionnaire. Malgré la présence de ressources économiques et de compétences, on voit à Esmeraldas comment la ressource pétrolière contribue à alimenter la fabrique des risques sur le territoire et dans la durée par la production directe de danger mais aussi par la désorganisation de la gouvernance.

\section{Conclusion}

La ville d'Esmeraldas présente de prime abord de nombreux paradoxes, en particulier la localisation d'enjeux majeurs pour l'économie et d'enjeux politiques autour de la souveraineté nationale dans un lieu fortement exposé aux séismes et aux tsunamis. Mais ç'en est un autre, plus connu sous l'appellation de la malédiction des ressources ou du syndrome hollandais, qui retient notre attention ici. L'analyse désormais classique de la conjonction d'une ressource lucrative et de formes aigües de pauvreté ou de gouvernance extrêmement problématique laisse penser qu'une telle situation n'a, sur le fond, rien de "paradoxal». De toute évidence, la ressource pétrole, seule, n'est fatalement porteuse d'aucune conséquence, ni pour le territoire, ni pour la gestion des risques (le cas de la Norvège devrait toujours contraster le fatalisme que véhicule le syndrome hollandais). Les reconfigurations de la gouvernance des risques et des dynamiques territoriales à Esmeraldas, et la différenciation des multiples acteurs en présence s'inscrivent dans des contextes sociaux, politiques, institutionnels, historiques, économiques ou géostratégiques. Si nous n'en avons développé que quelques aspects dans l'étude des relations entre risques, ressources et territoire à Esmeraldas à travers la gouvernance, c'est pour souligner le caractère éminemment contextuel de l'évolution des ressources et, surtout, de leur gestion 
sur les territoires, et pour évoquer la possibilité de trajectoires alternatives à celles que l'on repère sur la période récente au regard des risques. Une fois de plus, la géographie des risques invite à une analyse géopolitique.

En 2017, un comité national de gestion des risques liés aux hydrocarbures a été mis en place au niveau national. Le secteur des hydrocarbures semble commencer à prendre la mesure de ses lacunes dans la gestion des risques du fait d'une forme de déterritorialisation et d'une lecture trop exclusivement sectorielle des risques. Dans le même temps, et au-delà des premières confrontations entre la nouvelle mairie d'Esmeraldas et PetroEcuador, l'administration municipale fraîchement installée s'est montrée très désireuse de pouvoir «mutualiser » des compétences (mobiliser les compétences des acteurs pétroliers, ou de la coopération, mieux dotés) tout en cherchant à se positionner directement sur les nombreux investissements (de l'État ou des acteurs du pétrole) qui interviennent sur le territoire municipal et qui échappent à son autorité. C'est moins l'absence de financements et de compétences qui font problème à Esmeraldas que la coordination de leur usage.

Ainsi, à partir d'une lecture territoriale ancrée dans la littérature du risque (D'Ercole et Metzger, 2009), et sur la base d'une éventuelle convergence d'intérêts entre les acteurs d'une gouvernance des risques et du territoire jusque-là très fragmentée, il pourrait être utile de rendre visible des enjeux communs, ou tout du moins des formes d'interdépendances, de façon à œuvrer conjointement sur le territoire, vers moins de risques pour tous. Sans verser dans des recommandations idéalistes, cette perspective souligne le rôle que peuvent jouer les sciences sociales, éventuellement en interaction avec les acteurs gestionnaires, à travers des interprétations du monde social et une lecture du territoire, qui articulent risques et ressources.

\section{Bibliographie}

Acosta A. M., Albornoz V., 2010. «Rente pétrolière et politique budgétaire en Équateur ». Revue internationale de politique comparée, 17 (3) (p.111-126)

Burgos Cisneros A., 2016. La contaminación invisibilizada. Percepciones, representaciones y discursos sobre la contaminación ambiental en La Oroya y Esmeraldas. Mémoire de Maestría, Quito, FLACSO (168 p.)

Castillo J.D., 2011. Album de recortes de Esmeraldas. Archives de la bibliothèque municipale d'Esmeraldas (106 p.)

CEPAL, 1990. Impacto ambiental de la contaminación hídrica producida por la refinería estatal Esmeraldas: Análisis técnico-económico. Estudios e Informes de la CEPAL \# 80, Santiago de Chile (208 p.)

Collin Delavaud A., 2018. Les dynamiques territoriales des régions côtières de l'Équateur au XXe siècle. Paris, L'Harmattan (430 p.) 
Collin Delavaud A., 1978. « Esmeraldas, un foyer industriel national dans une zone forestière non intégrée ", in Foyers industriels nouveaux en Amérique latine, Paris, Editons IHEAL (p. 169-180)

CONADE, 1980. El estrato popular urbano de la ciudad de Esmeraldas. Quito, Consejo Nacional de Desarrollo

D'Ercole R., Metzger P., 2009. «La vulnérabilitéterritoriale: une nouvelleapproche des risques en milieuurbain ». Cybergeo : European Journal of Geography [En ligne], http://journals.openedition.org/cybergeo/22022.

Dalmasso E., Fillon P., 1970. «Influences comparées de Quito et Guayaquil (Équateur) ». Bulletin de l'Association de géographes français, volume 47, n³82-383 (p. 213-21)

Dourlens C., Galand J.-P., Theys J., Vidal-Naquet P., 1991. Conquête de la sécurité, gestion des risques, Paris, L'Harmattan (300 p.)

Estupiñán E., 2004. Municipio y sociedad en la lucha contra la desigualdad», in FLACMA, El libro del poder, Tomo 4 (p. 115-117)

Estupiñán J.P., 1975. «Esmeraldas en marcha ». Revista de la municipalidad de Esmeraldas, con la colaboración del H. Consejo Provincial. Archives de la bibliothèque municipale d'Esmeraldas (69 p.)

Estupiñán Carvache M., 2011. La segregación urbana en la Ciudad de Esmeraldas. Mémoire de Maestría, Quito, FLACSO (157 p.)

Fontaine G., 2015. «El paradigma perdido: la iniciativa Yasuní-ITT como falla de política ». Sociedad Suiza de Americanistas, $\mathrm{n}^{\circ} 76$ (p. 115-128)

Fontaine G., 2006. Petróleo y desarrollo sostenible en Ecuador, Vol 3. «Las ganancias y pérdidas », Quito, FLACSO (368 p.)

Fontaine G., Narváez I., 2007. Yasuní en el siglo XXI. El estado ecuatoriano y la conservación de la Amazonía. Travaux de 1'IFEA, n² 249, Lima (341 p.)

GADME, 2015. PDOT actualizado - Plan de ordenamiento territorial 2015-2022

GADME, 2012. PDOT - Plan de ordenamiento territorial 2012-2022

GADME-OXFAM, 2012. Estrategia de Gestión de riesgos y desastres. Cantón Esmeraldas. Esmeraldas, GADME, OXFAM, ECHO, SNGR (41 p.)

García-Acosta V., 2005. «El riesgo como construcción social y la construcción social del riesgo ». Desacatos, $\mathrm{n}^{\circ} 19$ (p. 11-24)

Gilbert C., 2013. "Quels risques pour la recherche en scienceshumaines et sociales », in Bourg D., Joly A. et Kaufman P-B., Du risque à la menace. Penser la catastrophe, Paris, PUF, (p. 219236) 
Harari R., Harari F., Forastiere F., 2016. « Environmental nickel exposure from oil refinery emissions: a case study in Ecuador ». Annali dell'Istituto Superiore di Sanità, Vol. 52, n 4 (p. 495-499)

Jaramillo M., 1980. Diagnóstico socio-económico de la Provincia de Esmeraldas. 3 vol. Gallocapitán, Otavalo

Jurado J., 2006a. « El petróleo como fuente de conflicto ambiental urbano: Esmeraldas bajo la influencia de una refinería ", in Fontaine G., Petróleo y desarrollo sostenible en Ecuador, Vol. 3, Quito, FLACSO (p. 169-187)

Jurado J., 2006b. "Esmeraldas: Los planes de manejo para 'enfrentar al monstruo' ", in Fontaine G., Petróleo y desarrollo sostenible en Ecuador, Vol. 3, Quito, FLACSO (p. 337-353)

Juteau-Martineau G., Becerra S., Maurice L., 2014. « Ambiente, petróleo y vulnerabilidad política en el oriente ecuatoriano: ¿Hacia nuevas formas de gobernanza energética? ». América Latina Hoy, $\mathrm{n}^{\circ} 67$ (p. 119-137)

Le Quang M., Ramìrez Gallegos F., 2016. «Introduction. L’Équateur de Rafael Correa : transition postnéolibérale et conflictualité ». Cahiers des Amériques Latines, ${ }^{\circ}$ 83, Paris, (p. 17-32)

Maurice L., 2018. Présentation du RapportMonoil - Monitoreo ambiental, salud, sociedad y petróleo en el Ecuador. Quito

Minda P., 2015. «La construcción del sujeto histórico afrodescendiente en Esmeraldas (Ecuador), siglos XVI y XIX ». Nova vetera, $\mathrm{n}^{\circ} 24$ (p. 5-17)

OIPE,1975. Propuesta de ordenamiento urbano y cantonal de Esmeraldas. Tome 2. Municipalité et province d'Esmeraldas (p. 100-184)

Oliver-Smith A., Alcántara-Ayala I., Burton I., Lavell A., 2017. " The social construction of disasterrisk: Seekingroot causes ». International Journal of Disaster Risk Reduction, $\mathrm{n}^{\circ} 22$, Editorial (p. 469-474)

OMC, 2019. Fiche par pays membre, 2017

https://www.wto.org/english/res_e/statis_e/daily_update_e/trade_profiles/EC_e.pdf

Pacheco Luque J. A., 2011. Contaminantes industriales y salud. Incidencia en la salud de los moradores del barrio 15 de Marzo, de los contaminantes gaseosos, emitidos por la refinería de petróleos y TermoEsmeraldas, en el periodo 2005-2010. Diplôme supérieur en gestion intégrale des risques et désastres, Quito, IAEN (76 p.)

Perrin J.-L., Janeau J.-L., Podwojewski P., 1998. Deslizamientos de tierra, inundaciones y flujos de lodo en Esmeraldas; diagnostico general de la situación actual en la ciudad, misión de expertos. Quito, Informe ORSTOM (21 p.) 
Pigeon P., Rebotier J., 2016. Disaster Prevention Policies. A Challenging and Critical Outlook, Londres, ISTE-Elsevier (240 p.)

Ramírez Gallegos F., 2010. « Post-neoliberalismo indócil. Agenda pública y relaciones socioestatales en el Ecuador de la revolución ciudadana ». Temas y Debates, $n^{\circ} 20$ (p. 175-194).

Rebotier J., 2016. El riesgo y su gestión en Ecuador: una mirada de geografía social y política. Quito, Pontificia Universidad Católica del Ecuador (145 p.)

Rebotier J., 2015. «Politiques de gestion des risques en Équateur. Entre coopération internationale et réaffirmation de l'État ». Autrepart, Vol. 2-3, n 74-75 (p. 279-295)

Rebotier J., Pigeon P., Metzger P., 2019. «Returning social context to seismic risk knowledge \& management. Lessons learned from an interdisciplinary research in the city of Esmeraldas, Ecuador ». Cybergeo: European Journal of Geography [En ligne], https://journals.openedition.org/cybergeo/31787

Rueda Novoa R., 2001. «Esclavos y negros libres en Esmeraldas s. XVIII-XIX ». ProcesoS, $\mathrm{n}^{\circ} 16$ (p. 3-33)

Sandoval Moreano J.F., 1990. Petróleo y desarrollo regional en Esmeraldas, CEPAL (208 p.)

Serrano T., D'Ercole R., 2003. « Análisis y cartografía de las amenazas de origen natural en la provincia de esmeraldas », in Amenazas, vulnerabilidad, capacidades y riesgo en el Ecuador. Los desastres, un reto para el desarrollo, COOPI-IRD-OXFAM (225 p.)

Torres F., 2000. Tiwintza el fin de un conflicto, pasado y presente del problema territorial Ecuador-Peru. Quito, AbyaYala (131 p.)

Valdivia G., 2018. "'Wagering Life' in the Petro-City: Embodied Ecologies of Oil Flow, Capitalism, and Justice in Esmeraldas, Ecuador». Annals of the American Association of Geographers, Vol. 108, Issue 2 (p. 549-557)

Wisner B., Blaikie P., Cannon T., Davis I., 1994. At Risk: natural hazards, people's vulnerability and disasters (Second edition 2003). New York, Routlege (471 p.) 